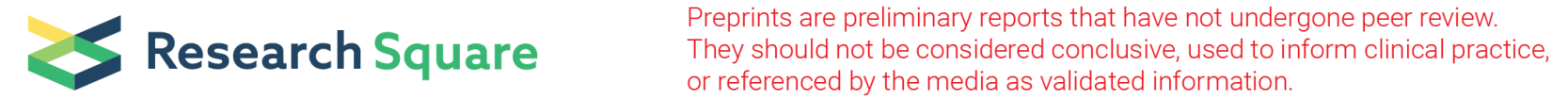 \\ Application of Multidisciplinary Intervention Model in Nursing of Older patients with Diabetes
}

\section{Zhonghong Zhao}

The second hospital of shandong university

Xiaoyun Wang ( $\nabla$ xywwangxiaoyun@163.com )

The second hospital of shandong university

\section{Research article}

Keywords: Multidisciplinary Intervention Model, Nursing, Older patients, Diabetes, Prognosis, Life quality

Posted Date: November 13th, 2019

DOI: https://doi.org/10.21203/rs.2.17174/v1

License: (9) This work is licensed under a Creative Commons Attribution 4.0 International License. Read Full License 


\section{Abstract}

Objective To evaluate the application of multidisciplinary treatment (MDT) model in nursing of elderly patients with diabetes.

Methods A total of 166 elderly diabetic patients were retrospectively selected. They were randomly and equally divided into the MDT group and the control group. The patients in the control group were treated with the traditional nursing method. And the patients in the MDT group were treated with a multidisciplinary team. The hospitalization time, hospital costs, incidence of diabetes-related complications, and nursing satisfaction were analyzed and compared.

Results The hospitalization time and hospitalization costs were significantly lower in the MDT group than those in the control group $(P<0.05)$. The satisfaction rate of MDT group $(98.3 \%)$ was significantly higher compared with the control group $(86.6 \% ; \mathrm{P}<0.05)$. Besides, the incidence of complications was significantly lower in the MDT group $(10.8 \%)$ when comparing to the control group $(40.1 \%$; $P<0.05)$.

Conclusion The application of MDT model in elderly diabetic care was superior to traditional routine nursing model, shortening the hospital stay and hospitalization costs, decreasing the incidence of complications and improving the nursing satisfaction of patients and their families, thereby improving the long-term prognosis and life quality of elderly diabetic patients.

\section{Introduction}

Diabetes is the most common metabolic disease due to impaired insulin secretion and varying degrees of insulin resistance, the main clinical manifestation of diabetes [1] [2]. Now diabetes is a frequently occurring disease without cure, especially in the older people [3, 4]. The older patients with diabetes often develop diabetic foot, cerebral infarction, diabetic retinopathy, etc, seriously affecting the their life quality $[5,6]$. Moreover, the clinical studies have also shown that older patients with diabetes often suffer from mental stress, anxiety, urgency, fear, loneliness, despair, depression or excitement, increasing the levels of pathology [7-9]. Therefore, the psychological and social factors play a critical role in the occurrence and development of diabetes, rendering psychological care as an important therapy of older diabetic patients. As reported, the different psychological treatments of older patients with different nursing interventions can improve the metabolic control of diabetes, improving the quality of life of patients [10-12].

Multidisciplinary treatment (MDT) model was originally introduced in the 1990s for the cancer treatment [13]. In 2007, the MDT model was also recommended to be used in the treatment of patients with type 2 diabetes $[14,15]$. The MDT model has been reported to provide the greatest positive effect on glycemic control in diabetic patients [16]. Graeme J Poston, the president of the European Society of Tumor Surgeons, introduced the MDT concept in China, and it has become a major trend in the treatment of metabolic diseases in China [17]. 
The MDT model in the comprehensive treatment of older diabetes has become an inevitable trend in medical development. Nursing, as an important part of multidisciplinary team collaboration, directly affects the prognosis and rehabilitation of patients [18]. The long-term treatment of patients led to many problems, including the changes of lifestyle, worriness about the complications, increased economic burden and other adverse factors, seriously affecting the treatment of patients. As expected, routine care intervention alone was difficult to achieve desired effects $[19,20]$. Therefore, the application of multidisciplinary intervention (MDI) model in the clinical care of older patients with diabetes is necessary.

In this study, to evaluate the application of MDI model in nursing of older patients with diabetes, the hospitalization time, hospital costs, incidence of diabetes-related complications, and nursing satisfaction were analyzed and compared between the patients treated with the conventional nursing method and a multidisciplinary team.

\section{Methods}

\section{Patients}

This study was approved by the Ethics Committee of the Second Hospital of Shandong University, and written informed consent was obtained from all patients. All methods were performed in accordance with the relevant guidelines and regulations.

A total of 166 older patients with diabetes in the Second Hospital of Shandong University between January 2013 and January 2015 were studied. The patients were randomly divided into two groups, including the MDI group ( $n=83)$ and the control group $(n=83)$, with 92 males and 74 females. The patients' basic information was listed in Table 1.

Inclusion criteria: Patients older than age 60 years; patients diagnosed with type 2 diabetes combined with chronic ulcers, cancer, chronic bronchitis, coronary heart disease, high blood pressure and other chronic diseases; patients without visual and hearing impairment in verbal communication; patients with certain self-care ability.

Exclusion criteria: Patients with disturbance of consciousness; patients with severe infection, diabetic ketoacidosis, heart failure, and other acute or serious diseases that seriously affect the study; patients with organic psychosis or long-term use of anti-anxiety and anti-depression drugs; patients with other cardiovascular and cerebrovascular diseases.

\section{Intervention}

The patients in the control group received routine nursing care, namely, the diabetic patients admitted to the department were treated according to the clinical nursing path of the department and the experience of the specialist nurse. The main nursing contents included assisting with admission procedures, 
introducing departments, monitoring routine life indicators, teaching health related knowledge, conducting psychological counseling, guiding medication, guiding diet, and assisting in rehabilitation exercises, etc.

The patients in the MDI group received nursing intervention caring by a multidisciplinary team, consisting of the specialist nurses in diabetes, geriatrics, angiocardiopathy, nutrition, and neurology, with 5 years of specialist experience and specialist nurse qualification. The collaborative team was formed within 30 min after patients diagnosed. The procedures were listed as follows: within 30 min of patients' hospital admission, each specialist proposed the problems faced by the patients in various fields. Then, the responsible nursing staff was responsible for the admission assessment and the establishment of nursing medical records. Next, different interventions were used for different patients. Diabetes specialist nurses performed the diabetes care in diabetic patients, such as the existence of diabetic foot, microcirculation disturbance, hypoglycemia, bad diet habits, fundus lesions, etc. Geriatric nurses developed older disease-related care programs, including swallowing function assessment and training, as well as bedridden patients' physical exercise and lung function training. Cardiovascular nurses assessed the cardiovascular functions of patients, like high blood pressure classification and cardiac function classification, and provided the examination-related guidance, cardiovascular health education, and secondary prevention work. Nutrition nurse assessed the nutritional status of patients, guiding patients to balance the diet, such as the diabetes diet, glycemic syndrome diet, and catering. The patients were also referred to the department of neurology for the diabetic neuropathy, such as the limb function determination, providing the guidance for choosing the method of peripheral nerve function exercise.

\section{Outcome variables}

After intervention, the hospitalization time, hospital costs, the incidence of diabetes-related complications and nursing satisfaction were analyzed and compared between the control group and the MDI group.

Nursing satisfaction was investigated by anonymous questionnaire using a self-made questionnaire. The total score was 100 points and the score above 80 points was considered as satisfactory. The incidence of complications such as partial pressure sores, lung infection, hip fracture, hyperglycemia and hypoglycemia were counted.

\section{Statistical analysis}

Statistical analysis was performed using the SPSS 23.0 statistical software. The measurement data were shown as mean \pm standard deviation and the difference between the two groups was analyzed using the independent samples t-test or Mann-Whitney rank sum test. The enumeration data were analyzed by the chi-square $(\chi 2)$ test or Fisher's exact probability analysis. $P$-values less than 0.05 were considered as significantly different. 


\section{Results}

\section{Comparison of patients' basic information between control and MDI groups}

There was no statistically significant difference in the age, gender, and disease course between the control group and the MDI group ( $P>0.05$; Table 1$)$.

\section{Comparison of hospitalization time, hospitalization costs and patient satisfaction between control and MDI groups}

As shown in Table 2, the hospitalization time in the MDI group ( $8.2 \pm 1.7$ days) was significantly lower than that in the control group $(14.1 \pm 2.7$ days; $P<0.05)$. Similarly, the hospitalization costs in the MDI group (39635.7 \pm 6752.1 Chinese Yuan) were significantly lower than those in the control group (49899.8 \pm 9367.5 Chinese Yuan; $P<0.05)$. Besides, the satisfaction rate of the MDI group (98.8\%) was significantly higher than that of the control group $(86.7 \% ; P<0.05)$.

\section{Comparison of complications in patients of the two groups}

After intervention, the incidence of complications in the control group was $41 \%$ (34 out of 83 cases), including 7 cases of partial pressure sores, 3 cases of lung infection, 4 cases of hip fracture, 11 cases of hyperglycemia and 9 cases of hypoglycemia. The incidence of complications in the MDI group was $9.6 \%$ ( 8 out of 83 cases), including 2 cases of partial pressure sores, 2 cases of hip fracture, 3 cases of hyperglycemia and 1 case of hypoglycemia. As statistically analyzed, the difference in the incidence of complications after intervention between the two groups was statistically significant $(P<0.05)$ (Table 3).

\section{Discussion}

In the comprehensive treatment of diabetes mellitus, the change of medical model and the improvement of psychological expectation of patients put forward higher requirements for nursing work [19, 21]. The staffs in daily routine nursing should pay more attention to the psychological needs of patients, as psychological care for older diabetic patients with anxiety and depression could significantly improve the quality of life [10-12]. Besides, the patients with dietary guidance and treatment considerations should be given the psychological intervention and health education to enhance the treatment compliance.

Therefore, the establishment of a platform that implements the comprehensive nursing intervention, 
including the health education, psychological intervention, regular medical and patient exchange activities, family social support as well as various types of routine care, is of great significance.

Studies have shown that the MDI model could be applied in the nursing practice of patients with a variety of diseases to strengthen the personalized care of patients, avoid unnecessary expenses, reduce the patients' hospital costs, and decrease the patients' economic pressure to a certain extent [17]. In our study, the application of the MDI model in older diabetic care provided better outcomes than the traditional routine nursing model. As shown, we found that the MDI model used in older diabetes care could significantly reduce the average hospital stay and hospital costs, and improve the satisfaction of patients. Especially, the hospitalization costs in the MDI group (39635.7 \pm 6752.1 Chinese Yuan) were significantly lower than those in the control group (49899.8 \pm 9367.5 Chinese Yuan; $P<0.05)$; similar results were observed in the specialized Multidisciplinary Community-Based Care for diabetic patients, not restricted to older patients [22]. Compared with the control group, patients in the MDI group received more targeted guidance from more specialist caregivers, and the diabetes-related complications were further controlled, with the cases affected by pulmonary infection, hip fractures, local bedsores, hyperglycemia, hypoglycemia and other complications being significantly reduced ( $9.6 \%$ vs. $41 \%$ ), consistent with previous study [23]. Thus, the application of the MDI model can improve the long-term prognosis and quality of life of older diabetic patients, and the development of MDI model in the care for older patients with diabetes is a guiding significance.

The care programs of a multidisciplinary collaborative care model for hospitalized diabetic elders were established by centralized consultation, reducing the repeated nursing consultation time of older diabetic patients with multiple diseases and thus effectively shortening the hospital stay [24, 25]. In the multidisciplinary team teamwork model, all nursing staffs have a new and improved understanding in the care theory of older patients with diabetes through business study, pathological discussion, bedside rounds, and head nurse on-site management [7, 26-28]. Through the multi-pronged cooperation, the overall level of disease management around patients has been raised. Meanwhile, both the hospital health education and out-patient health education play a very important role in the establishment of management rules that the nurse managers and faculty should observe, not only limited to specialist nursing. Moreover, the nurse managers and faculty coordination could greatly reduce the workload and improve the work progress of staff, rapidly improving the outcomes of the patients. And students can also learn a wide range of nursing knowledge, making students' progress more clearly in the learning process. However, we still need to focus on the construction and training of specialist care teams, developing detailed training programs, improving and optimizing the perioperative management process and quality standards, adapting to the new situation of medical activities. It should be mentioned that the patients' safety and interests should always be in the first place during the design of scientific and rational personalized care plan.

However, there were several limitations in our study. First, the sample size was small, requiring more samples for further validation. Second, the MDI in our hospital consisted of only several departments, possibly resulting in some deviations. Finally, the quality of care and the knowledge level of nursing staff 
should be improved. Therefore, it is urgent to ameliorate the professional technical and cultural level of nursing staff. Besides, we will continue to collect a large amount of data, verifying the repeatability and authenticity of our findings. Further, we will expand our research direction, focus on certain types of patients, and refine the specialist nursing details, hoping to achieve the shortest possible time with the best departments and the least staffs, and the most reasonable service, improving the patients' prognosis.

In conclusion, the application of MDI model in older diabetic care was superior to traditional routine nursing model, reducing the hospital stay and hospitalization costs, decreasing the incidence of complications, improving the nursing satisfaction of patients and their families, and thereby improving the long-term prognosis and life quality of older diabetic patients. Therefore, MDI could be used in the care of older diabetes to ensure the patients receive the most appropriate treatments and rehabilitation programs, countering the defects of a single care model.

\section{Declarations}

\section{Abbreviations}

Multidisciplinary treatment (MDT)

Multidisciplinary intervention (MDI)

\section{Ethics approval and consent to participate}

All procedures performed in this study were in accordance with the ethical standards of The Second Hospital of Shandong University and with the 1964 Helsinki declaration and its later amendments or comparable ethical standards.

\section{Consent for publication}

Written informed consent was obtained from all participants.

\section{Availability of data and material}

The analyzed data sets generated during the study are available from the corresponding author on reasonable request.

\section{Competing interests}

There are no potential conflicts with any person or organization regarding this study.

\section{Funding}


None

\section{Authors' contributions}

Conception and design: ZHZ.

Analysis and interpretation of data: XYW.

Drafting the article: $\mathrm{ZHZ}$

Revising it critically for important intellectual content: XYW.

GUARANTOR: XYW.

\section{Acknowledgements}

Not applicable.

\section{References}

1. Escalada, J., et al., Attitudes towards insulin initiation in type 2 diabetes patients among healthcare providers: a survey research. Diabetes research and clinical practice, 2016. 122: p. 46-53.

2. Furler, J., et al., Supporting insulin initiation in type 2 diabetes in primary care: results of the Stepping Up pragmatic cluster randomised controlled clinical trial. bmj, 2017. 356: p. j783.

3. Wang, Q., et al., Prevalence, awareness, treatment and control of diabetes mellitus among middleaged and elderly people in a rural Chinese population: A cross-sectional study. PloS one, 2018. 13(6): p. e0198343.

4. Zhao, C., et al., Prevalence and correlates of chronic diseases in an elderly population: A communitybased survey in Haikou. PloS one, 2018. 13(6): p. e0199006.

5. Camacho, E.M., et al., Long-term cost-effectiveness of collaborative care (vs usual care) for people with depression and comorbid diabetes or cardiovascular disease: a Markov model informed by the COINCIDE randomised controlled trial. BMJ open, 2016. 6(10): p. e012514.

6. Hsieh, Y.-L., et al., Factors Influencing Intention to Receive Examination of Diabetes Complications. Asian nursing research, 2016. 10(4): p. 289-294.

7. Tada, Y., et al., Long-term Efficacy and Safety of Sitagliptin in Elderly Patients with Type 2 Diabetes Mellitus. Intern Med, 2016. 55(10): p. 1275-8.

8. Kukidome, D. and E. Araki, [Key notes of glycemic control in elderly patients with diabetes]. Nihon Rinsho, 2016. 74 Suppl 2: p. 551-5.

9. Nourizadeh-Sedaghati, A., et al., Study of Insulin Requirement Modeling in Hospitalized Elderly Patients with Type 2 Diabetes at a Late Stage of Stepwise Escalation Therapy. Diabetes Technol Ther, 2016. 18(5): p. 308-15.

10. Targonski, R., J. Sadowski, and K. Zajac, Comment on Modifying Effect of Body Mass Index on Survival in Elderly Type 2 Diabetic Patients: Hong Kong Diabetes Registry and Reverse Epidemiology 
of Traditional Cardiovascular Risk Factors in the Geriatric Population. J Am Med Dir Assoc, 2016. 17(6): p. 561-2.

11. van Dijk, P., et al., Hypoglycemia in Frail Elderly Patients With Type 2 Diabetes Mellitus Treated With Sulfonylurea. J Diabetes Sci Technol, 2017. 11(2): p. 438-439.

12. Chin, S.O., et al., Hypoglycemia is associated with dementia in elderly patients with type 2 diabetes mellitus: An analysis based on the Korea National Diabetes Program Cohort. Diabetes Res Clin Pract, 2016. 122: p. 54-61.

13. Bradley, P.J., Multidisciplinary clinical approach to the management of head and neck cancer. Eur Arch Otorhinolaryngol, 2012. 269(12): p. 2451-4.

14. Care, F., Standards of medical care in diabetes-2017. Diabetes Care, 2017. 40(Suppl 1): p. S88-98.

15. Members, A.T.F., et al., ESC Guidelines on diabetes, pre-diabetes, and cardiovascular diseases developed in collaboration with the EASD: the Task Force on diabetes, pre-diabetes, and cardiovascular diseases of the European Society of Cardiology (ESC) and developed in collaboration with the European Association for the Study of Diabetes (EASD). European heart journal, 2013. 34(39): p. 3035-3087.

16. Shojania, K.G., et al., Effects of quality improvement strategies for type 2 diabetes on glycemic control: a meta-regression analysis. Jama, 2006. 296(4): p. 427-440.

17. Yu, J.C., et al., Application of laparoscopic adjustable gastric banding and a multi-disciplinary team approach in treatment of morbid obesity and its complications. Acta Academiae Medicinae Sinicae, 2011. 33(3): p. 265-271.

18. Ryan, J. and I. Faragher, Not all patients need to be discussed in a colorectal cancer MDT meeting. Colorectal Dis, 2014. 16(7): p. 520-6.

19. Grohmann, B., S. Espin, and E. Gucciardi, Patients' experiences of diabetes education teams integrated into primary care. Can Fam Physician, 2017. 63(2): p. e128-e136.

20. Kezerle, L., L. Shalev, and L. Barski, Treating the elderly diabetic patient: special considerations. Diabetes Metab Syndr Obes, 2014. 7: p. 391-400.

21. Farmer, A.J., et al., The effect of a brief action planning intervention on adherence to double-blind study medication, compared to a standard trial protocol, in the Atorvastatin in Factorial with Omega EE90 Risk Reduction in Diabetes (AFORRD) clinical trial: A cluster randomised sub-study. Diabetes Res Clin Pract, 2016. 120: p. 56-64.

22. Specialized multidisciplinary community-based care series: a summary of evidence-based analyses. Ont Health Technol Assess Ser, 2009. 9(16): p. 1-23.

23. Jiao, F.F., et al., Five-Year Cost-effectiveness of the Multidisciplinary Risk Assessment and Management Programme-Diabetes Mellitus (RAMP-DM). Diabetes Care, 2018. 41(2): p. 250-257.

24. Utsunomiya, K., et al., Japanese study of tofogliflozin with type 2 diabetes mellitus patients in an observational study of the elderly (J-STEP/EL): A 12-week interim analysis. J Diabetes Investig, 2016. 7(5): p. 755-63. 
25. El-Tawdy, A.H., et al., Maggot Debridement Therapy (Mdt): It Is Safe and Economic for Treating a Diabetic Foot Ulcer. J Egypt Soc Parasitol, 2016. 46(1): p. 223-34.

26. Souza, J.G., et al., Applicability of the Spoken Knowledge in Low Literacy Patients with Diabetes in Brazilian elderly. Einstein (Sao Paulo), 2016. 14(4): p. 513-519.

27. Ma, L. and Y. Li, Cognitive function and insulin resistance in elderly patients with type 2 diabetes. Neurol Res, 2017. 39(3): p. 259-263.

28. Utsunomiya, K., et al., Safety and effectiveness of tofogliflozin in elderly Japanese patients with type 2 diabetes mellitus: A post-marketing study (J-STEP/EL Study). J Diabetes Investig, 2017. 8(6): p. 766-775.

\section{Tables}

Table 1. Comparison of patients' basic information between two groups

\begin{tabular}{lll}
\hline & Control & MDI \\
& $(\mathrm{n}=83)$ & $(\mathrm{n}=83)$ \\
\hline Age (years) & $72.2 \pm 6.6$ & $72.9 \pm 7.3$ \\
Gender (female/male) & $36 / 47$ & $38 / 45$ \\
\hline Disease course (years) & $12.1 \pm 3.2$ & $13.1 \pm 4.0$ \\
\hline
\end{tabular}

MDI, Multidisciplinary intervention.

Table 2. Comparison of hospitalization time, hospitalization costs and satisfaction rate between two groups

\begin{tabular}{lllll}
\hline Group & $\mathrm{n}$ & Hospitalization time (days) & Hospitalization costs (CNY) & Satisfaction rate \\
\hline Control & 83 & $14.1 \pm 2.7$ & $49899.8 \pm 9367.5$ & $72(86.7 \%)$ \\
MDI & 83 & $8.2 \pm 1.7^{*}$ & $39635.7 \pm 6752.1^{*}$ & $82(98.8 \%)^{*}$ \\
\hline
\end{tabular}

MDI, Multidisciplinary intervention; CNY, Chinese Yuan. *, $P<0.05$ vs. Control. 
Table 3. Comparison of complications in patients with diabetes mellitus

\begin{tabular}{|c|c|c|c|c|c|c|c|c|}
\hline Group & $\mathrm{n}$ & $\begin{array}{l}\text { Partial } \\
\text { sores }\end{array}$ & pressure & $\begin{array}{l}\text { Lung } \\
\text { infection }\end{array}$ & $\begin{array}{l}\text { Hip } \\
\text { fracture }\end{array}$ & Hyperglycemia & Hypoglycemia & Total \\
\hline Control & 83 & 7 (8.4\%) & & 3 (3.6\%) & $4(4.8 \%)$ & 11 (13.3\%) & 9 (10.8\%) & $\begin{array}{l}34 \\
(41.0 \%)\end{array}$ \\
\hline MDI & 83 & $2(2.4 \%)$ & & 0 & $2(2.4 \%)$ & $3(3.6 \%) *$ & $1(1.2 \%) *$ & $\begin{array}{l}8 \\
(9.6 \%)^{*}\end{array}$ \\
\hline
\end{tabular}

MDI, Multidisciplinary intervention. $*, P<0.05$ vs. Control. 